\title{
The role of large-scale vortical structures in transient convective heat transfer augmentation
}

\author{
David O. Hubble, Pavlos P. Vlachos $\dagger$ and Tom E. Diller \\ Department of Mechanical Engineering, Virginia Tech, Blacksburg, VA 24061, USA
}

(Received 6 November 2011; revised 24 November 2012; accepted 26 November 2012; first published online 8 February 2013)

The physical mechanism by which large-scale vortical structures augment convective heat transfer is a fundamental problem of turbulent flows. To investigate this phenomenon, two separate experiments were performed using simultaneous heat transfer and flow field measurements to study the vortex-wall interaction. Individual vortices were identified and studied both as part of a turbulent stagnation flow and as isolated vortex rings impacting on a surface. By examining the temporal evolution of both the flow field and the resulting heat transfer, it was observed that the surface thermal transport was governed by the transient interaction of the vortical structure with the wall. The magnitude of the heat transfer augmentation was dependent on the instantaneous strength, size and position of the vortex relative to the boundary layer. Based on these observations, an analytical model was developed from first principles that predicts the time-resolved surface convection using the transient properties of the vortical structure during its interaction with the wall. The analytical model was then applied, first to the simplified vortex ring model and then to the more complex stagnation region experiments. In both cases, the model was able to accurately predict the time-resolved convection resulting from the vortex interactions with the wall. These results reveal the central role of large-scale turbulent structures in the augmentation of thermal transport and establish a simple model for quantitative predictions of transient heat transfer.

Key words: turbulent convection, vortex flows, vortex interactions

\section{Introduction}

Predicting convective heat transfer in the presence of free-stream turbulence is difficult, even for simple geometries, because of the complex and random interactions of flow structures with the wall surface. Traditionally, methods for analysing the convective heat transfer in the presence of free-stream turbulence rely on average and statistical properties of the flow field. Such approaches result in empirical correlations and models that often are not based on the governing physics. For example, empirical correlations for the augmentation of time-average heat transfer by free-stream turbulence have been developed (Kestin 1966; Lowery \& Vachon 1975) based on statistical turbulence properties such as the turbulence intensity $(T u)$ and integral length scale ( $\Lambda$ ) (Vanfossen, Simoneau \& Ching 1995; Ames 1997). Mayle, Dullenkopf \& Schulz (1998) emphasized the importance of the turbulent spectrum 
and Dullenkopf \& Mayle (1995) identified a dominant frequency band corresponding to an eddy size that is about 16 times the laminar boundary layer thickness. A surface renewal model was used to estimate the effect of the turbulent structures from the free stream (Kataoka 1990; Yuan \& Liburdy 1992). Time-resolved experimental measurements of the fluctuating transport at the surface have shown direct correlation with the velocity fluctuations in the free stream (Moss \& Oldfield 1996; Holmberg \& Diller 2005), but the impact on the overall transport was not established. All of these models are valid only within the narrow range of the experimental data that yielded these correlations and do not offer a general predictive ability.

Adrian, Christensen \& Liu (2000) describe the role of proper decomposition of turbulent velocity fields depending on what is being studied. Although the statistics of the fluctuating fields may be useful for Reynolds-averaged models, visualization of the structured, coherent elements requires methods that show an entire section of the flow field as a function of time. For example, Hutchins \& Marusic (2007) showed that the inner boundary layer small-scale structure was directly influenced by the large-scale outer flow structures and in a later work they proposed a model capturing these interactions (Marusic, Mathis \& Hutchins 2010). The elegance of their model stems from its ability to predict the near-wall flow structure, which is very challenging to measure or accurately model, using large-scale information from the external flow. Although they focused on the turbulent statistics of the inner-region velocity and not the vortical flow structure, the connection they showed between the inner and outer regions as a function of time is most interesting and qualitatively supports the physical processes described here in the sense that the large-scale external flow governs the near-wall processes. The effect of turbulent spots on surface heat transfer was also studied by Sabatino \& Smith (2008) using a combination of particle image velocimetry (PIV) and liquid-crystal temperature measurements. By identifying the vortical structures within the boundary layer they demonstrated the importance of the temperature of the fluid that is brought to the surface for augmenting heat transfer.

Vortical structures are particularly important in stagnating flows where free-stream structures are aligned with the wall and amplified through vortex stretching by the mean flow strain rate (Sutera 1965). Gifford, Diller \& Vlachos (2011) used timeresolved digital particle image velocimetry (TRDPIV) and a thin-film heat flux sensor to study heat transfer augmentation in the stagnation region of a flow containing large-scale free-stream turbulence. Subsequent tracking of the vortices present showed that the rate of heat transfer at the wall was directly influenced by the strength and proximity of the vortex. It appeared that the largest transport occurred when the structures swept free-stream fluid through the boundary layer to the surface. This concept is supported by two numerical studies (Romero-Mendez et al. 1998; Martin \& Zenit 2008) of individual vortices near a surface and experimental measurements with synthetic jets (Pavlova \& Amitay 2006).

Other researchers have examined the time-average influence of vortices on surface transport. Vanfossen \& Simoneau (1987) used wires to create vortices perpendicular to a downstream cylinder and studied the resulting fluid flow and heat transfer near the surface using a combination of smoke visualization and liquid crystals. It was observed that regions where vortices forced fluid towards the surface corresponded to regions of increased heat transfer. Xiong \& Lele (2007) used large-eddy simulation (LES) to model isotropic turbulence impinging on a stagnation region. Vortices were observed to form near the stagnation boundary layer. These vortices had a stronger influence on the thermal transfer compared to the momentum transfer. Direct numerical simulation (DNS) was used to model the effect of turbulent structures on 
surface transport in channel flow (Araya, Leonardi \& Castillo 2008). Even when the structures are modelled directly, however, a turbulence model is still required to calculate the transport near the surface.

Finally, for investigating vortex-induced heat transfer augmentation, the vortex ring-wall interaction (Doligalski, Smith \& Walker 1994) provides an attractive system due to the inherent ability to control the flow characteristics with simplicity and reproducibility. Vortex rings have been extensively investigated and are very well understood from a fluid mechanics standpoint (Shariff \& Leonard 1992) but not much work is available considering vortex-ring flows in combination with heat transfer. In a recent experimental investigation (Arévalo et al. 2007) a vortex ring was created in air and allowed to impact on a heated vertical plate. The spatially averaged heat transfer from the surface was found to increase by up to $15 \%$ over natural convection levels while the vortex was in contact with the surface. In a subsequent study (Arévalo et al. 2010) they expanded their analysis by incorporating PIV measurements and quantifying the near-wall interaction and shear stresses. However, neither of these studies was concerned with the mechanism of the heat transfer augmentation driven by the vortex ring, and they did not attempt to resolve the spatiotemporal correlations between heat flux and the flow field.

In the present work we hypothesize that we can predict the near-wall transient heat transfer in a turbulent flow merely from the characteristics of the outer largescale vortical structures. To help clarify this approach we first apply our model to individual vortex rings striking the surface. This allows us to control the properties of the vortex and eliminates the complexity of multiple interacting vortices. The results with the vortex ring are then used in the more complex case of large-scale free-stream turbulence interacting with the surface. In both experiments, the timeresolved convective heat transfer is measured while TRDPIV is used to simultaneously measure the two-dimensional flow field. Analysing these measurements in combination reveals the mechanism by which the vortex structure augments the induced heat transfer. These observations then enable the development of an analytical model which predicts the transient convection based only on the properties of the large-scale vortical structure interacting with the heated wall. The predictive ability of the model is subsequently tested and validated, first with the simplified vortex ring and then with the stagnating turbulence data.

\section{Experimental measurements}

In this study two separate experiments were performed. In the first, the convection along the stagnation line of a bluff body in a flow which contains large-scale freestream turbulence is examined. In the second, a pneumatically actuated vortex ring generator is used to produce vortex rings which were allowed to collide normally with an instrumented heated surface. The two experiments share much of the experimental methodology as described in this section. In both experiments, measurements were made to resolve the flow field near the surface while simultaneously measuring the convection from the surface. Planar TRDPIV (Raffel et al. 2007) was used to measure the spatiotemporal characteristics of the flow field as it interacts with the surface. A vortex identification scheme (Zhou et al. 1999) was then used to identify and track the vortices present in the TRDPIV data. Measurements of the convective heat transfer coefficient at the surface were made using the newly developed TimeResolved Heat and Temperature Array (THeTA) (Hubble \& Diller 2010b). Convection measurements were performed at 10 equidistant points along the stagnation line in 


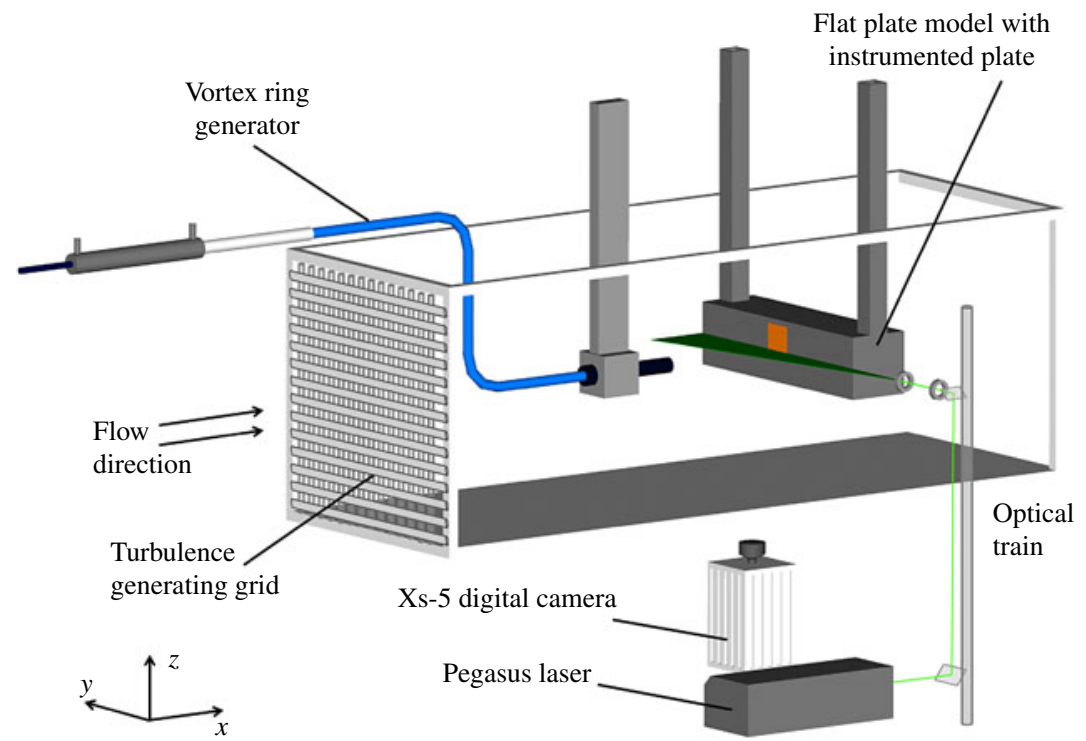

FIGURE 1. (Colour online) Water tunnel facility and experimental setup. During the stagnation tests the vortex ring generator was replaced with turbulence-generating grids.

the first experiment and a line that bisected the impinging vortex ring in the second experiment.

The experiments were conducted in the AEThER laboratory at Virginia Tech. The equipment used in the experiment includes: the water tunnel facility, a vortex ring generator, an instrumented heated plate, the TRDPIV system, and the accompanying data acquisition systems which also synchronized the heat flux and velocity measurements. A schematic of the overall setup is shown in figure 1. Note that the vortex ring generator is positioned normal to the flat plate and the height of the TRDPIV laser plane is such that it bisects the vortex ring generator's nozzle and aligns with the heat flux sensors on the heated plate. For the stagnation experiments, the vortex ring generator is replaced with turbulence-generating grids.

\subsection{Water tunnel facility and turbulence generation}

The water tunnel used in the current experiment is a closed loop, variable speed facility which was operated at $10 \mathrm{~cm} \mathrm{~s}^{-1}$ for all of the stagnation tests and was used as simply a water tank for the vortex ring tests. The test section measures $0.61 \mathrm{~m}$ square by $1.77 \mathrm{~m}$ long and is constructed from clear acrylic. Without turbulence grids and at the tunnel speed used in the stagnation tests the free-stream turbulence intensity is approximately $1 \%$ throughout the test section.

Free-stream turbulence was generated using simple bi-plane grids. Grid bar sizing $(b)$, spacing $(M)$ and positioning was based on correlations by Baines \& Peterson (1951). Three grids were constructed with solidity ratios of $S=0.7$ to give integral length scales $(\Lambda)$ of approximately 1,2 , and $3 \mathrm{~cm}$ at a turbulence intensity ( $\mathrm{Tu}$ ) of approximately $7 \%$. In all cases, the test region was located at a distance downstream of the grid equal to $17.5 \mathrm{M}$. Properties of the generated turbulence were calculated from the measured flow field with no stagnation model present at downstream distances $\leqslant 17.5 M$ and the results compared well with the published correlations 


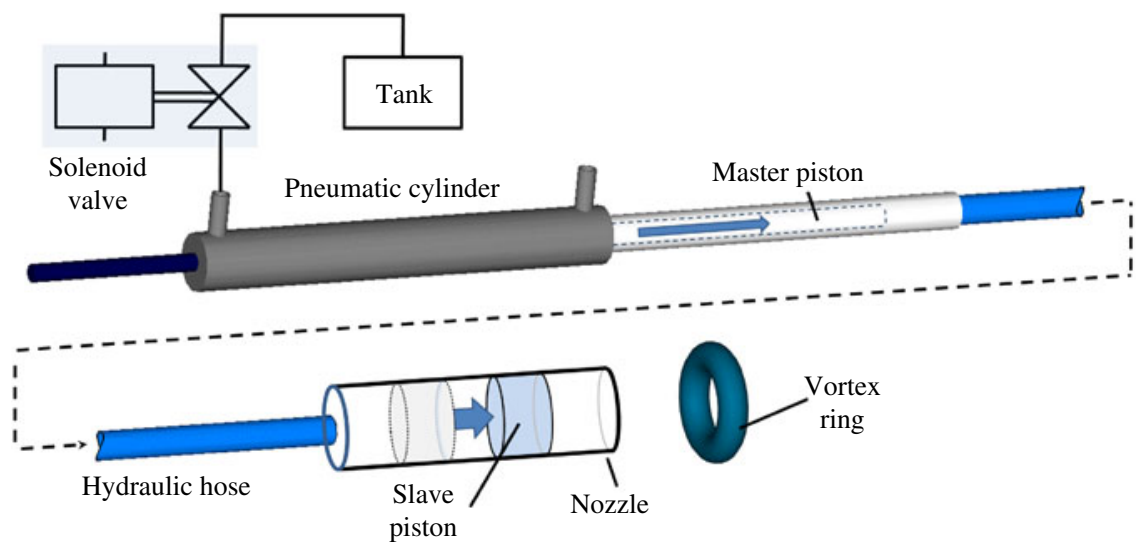

FIGURE 2. (Colour online) Schematic of the vortex ring generator.

$\begin{array}{lcccc}\text { Grid } & b(\mathrm{~cm}) & M(\mathrm{~cm}) & T u(\%) & \Lambda(\mathrm{cm}) \\ 1 & 0.66 & 1.46 & 7.4 & 0.81 \\ 2 & 1.60 & 3.54 & 7.2 & 1.93 \\ 3 & 2.16 & 4.77 & 6.9 & 3.24\end{array}$

TABLE 1. Details of grids and turbulence statistics.

(Baines \& Peterson 1951). These calculations were performed using the methods described by Gifford et al. (2011). Details of the grid geometry and turbulence properties are given in table 1. The turbulence intensity was kept almost constant (7\%) for all three length scales in order to focus on the effect of the turbulent length scale and the underlying heat transfer augmentation mechanisms.

\subsection{Vortex ring generator}

Vortex rings were produced in a conventional way (Shariff \& Leonard 1992) by ejecting a slug of fluid through a circular constant-diameter nozzle. The vortex ring generator is shown schematically in figure 2. A pneumatic cylinder is attached to a regulated air supply via a solenoid valve (MAC 224B). Prior to a test, the piston is withdrawn a prescribed distance corresponding to the desired piston stroke length. When the valve opens, air forces the piston to move the set distance to a stop and in doing so displaces a known volume of water. The displaced water travels through the hydraulic hose where it displaces the slave piston located inside the nozzle. Movement of the slave piston displaces the fluid in the nozzle which forms the vortex ring. The nozzle was manufactured from a clear acrylic tube with an inside diameter of $2.54 \mathrm{~cm}$. The transparency of the tube allowed the velocity history as well as the stroke length of the slave piston to be measured optically using a high-speed digital camera and standard shadowgraph techniques. Varying these parameters allowed the properties of the generated ring to be varied as shown in table 2 .

\subsection{Instrumented flat plate}

A basic flat-plate model was constructed as shown in figures 1 and 3. The model comprised a smooth, removable face plate mounted onto a watertight rectangular 


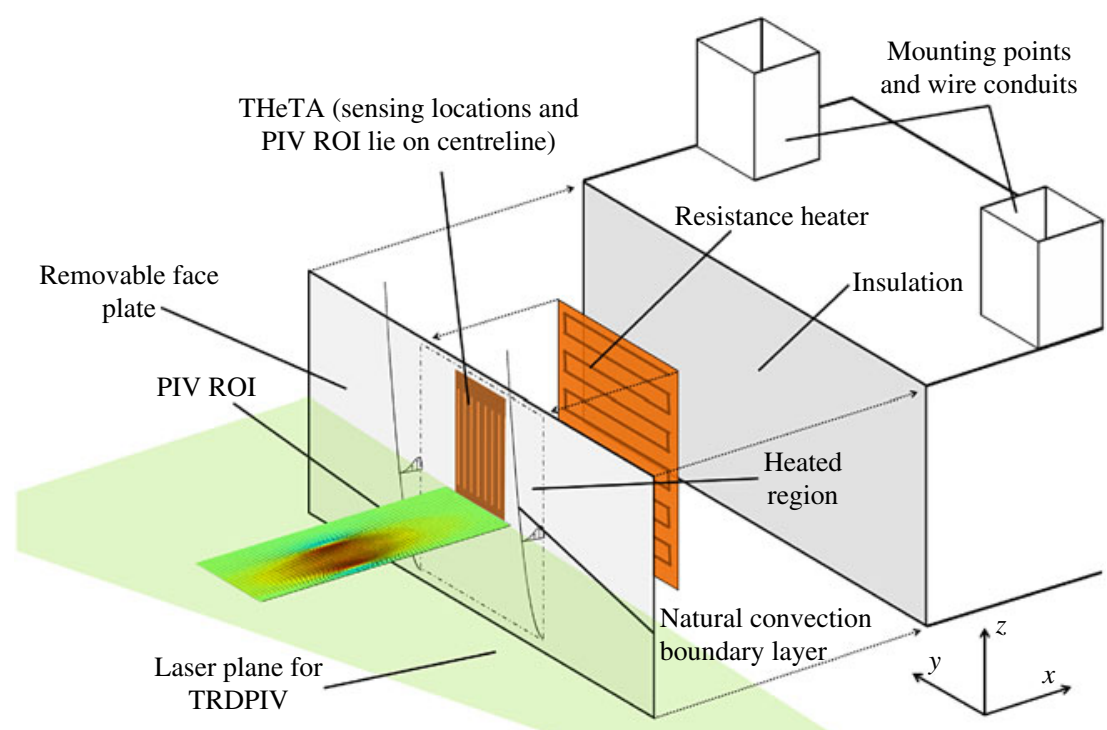

FIGURE 3. (Colour online) Flat-plate model and instrumentation. ROI denotes region of interest.

$\begin{array}{lcc}\text { Test } & R e=\Gamma / \nu & U\left(\mathrm{~cm} \mathrm{~s}^{-1}\right) \\ 1 & 3100 & 5.3 \\ 2 & 5400 & 8.8 \\ 3 & 5800 & 8.4 \\ 4 & 6300 & 9.9 \\ 5 & 9200 & 19.0\end{array}$

TABLE 2. Vortex ring experiment testing conditions: properties of the vortex rings.

housing which spanned the width of the water tunnel. Two long square tubes were welded to the housing and were used to hold the model rigidly and also served as conduits to carry power and signal wires out of the water tunnel. A $10 \mathrm{~cm}$ square in the centre of the $5 \mathrm{~mm}$ thick aluminium face plate was thermally isolated from the rest of the plate by a series of machined slots which were later filled with a low-conductivity silicone adhesive both to keep the model watertight and to keep the face plate smooth. A thin-film resistance heater rated at $1.5 \mathrm{~W} \mathrm{~cm}^{-2}$ (150 W total) was adhered to the back (inside) of the face plate and heated the test area approximately $30^{\circ}$ above the water temperature. Insulation was used on the back of the heater to force most of the heat through the face plate to be convected away by the water. Because of the thickness and high thermal conductivity of the plate $\left(175 \mathrm{~W}(\mathrm{~m} \mathrm{~K})^{-1}\right)$, the plate is maintained nearly isothermal in space and time during the tests.

Heat flux and surface temperature measurements were taken at ten locations along the centreline of the test area at a spacing of $6.3 \mathrm{~mm}$ using the THeTA, a polyimide (Kapton 100HN) based differential heat flux sensor. To minimize the thermal effect of the sensor's presence (less than $0.1 \mathrm{~mm}$ total thickness), the remainder of the heated section was masked with the same Kapton used in the sensor construction to maintain 


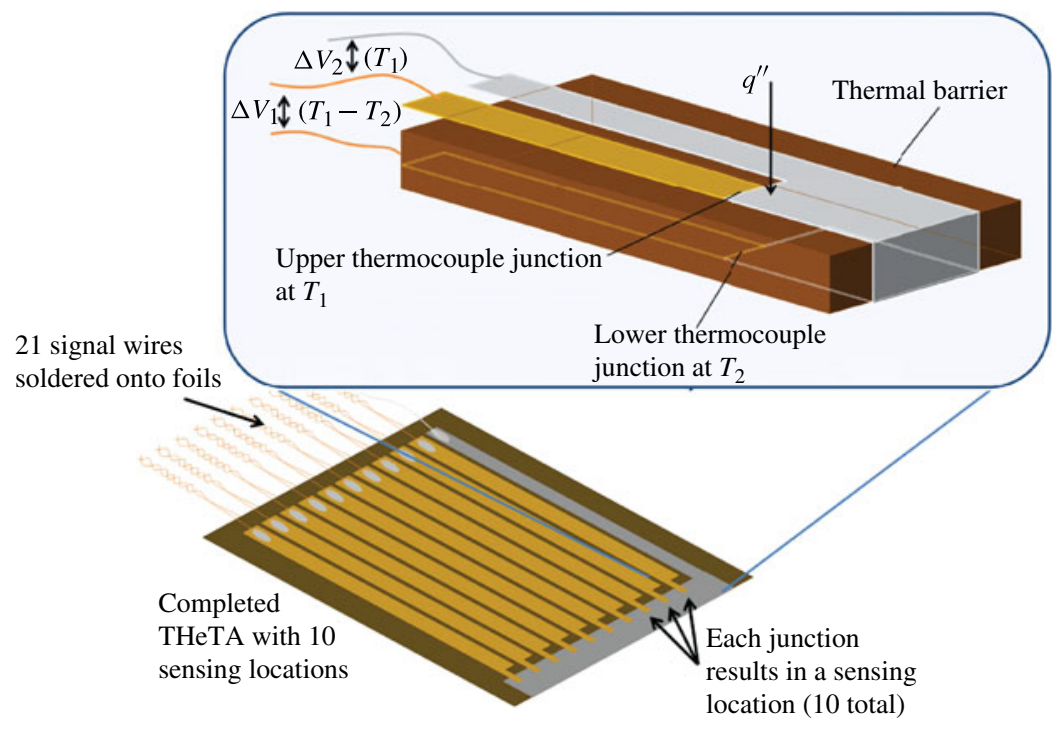

FIgURE 4. (Colour online) Schematic of the Time-resolved Heat and Temperature Array (THeTA).

uniform smooth surface conditions. Details of the development and calibration of the THeTA are given in Hubble \& Diller (2010b) and a depiction of the sensor is given in figure 4. Heat flux through the sensor is proportional to the temperature drop across the thermal barrier as directly measured by two type-T (copper-constantan) thermocouples connected in series $\left(\Delta V_{1}\right)$. The surface area of the thermocouple contact determines the effective measurement width, which was $1.6 \mathrm{~mm}$ or $25 \%$ of the sensor spacing. Surface temperature measurements are obtained from the thermocouple located on the top of the THeTA $\left(\Delta V_{2}\right)$. Each of the ten sensors on the THeTA was individually directly calibrated by conduction and radiation for the steady-state heat flux sensitivity and by radiation for the transient response, as described by Hubble \& Diller (2010b). Because of variations in the thickness during construction, the calibrated sensitivities of each sensor used are somewhat different. The average sensitivity of the ten heat flux sensors on the THeTA is $223 \mu \mathrm{V}\left(\mathrm{W} \mathrm{cm}^{-2}\right)^{-1}$ with a $95 \%$ time response of 509 ms. The Hybrid Heat Flux (HHF) (Hubble \& Diller 2010a) technique was used to improve the initial time response of the THeTA. The HHF is a numerical signal processing technique which increases the performance of differential heat flux sensors by accounting for the thermal energy stored within the sensor in conjunction with the heat flowing through the sensor. Differential sensors effectively low-pass filter the heat flux input due to the time required for heat to diffuse across their thickness. Utilizing the HHF method enables obtaining higher frequency information from the temporal derivative of the surface temperature signal, which is then added to the low-frequency information obtained from the temperature drop across the sensor. This method has been validated both numerically and experimentally and has been shown to improve the $95 \%$ time response of the THeTA to $36 \mathrm{~ms}$ (Hubble \& Diller 2010b).

The 20 signals from the THeTA (10 heat flux, 10 surface temperature) were sampled at a rate of $1 \mathrm{kHz}$ using a National Instruments 6225 16bit DAQ which also synchronized the TRDPIV camera and laser to the heat flux measurements 
and controlled the vortex ring generator when needed. The microvolt signals from the THeTA were first amplified using custom-fabricated amplifier boards (Ewing 2006) with a fixed gain of 1000 and $480 \mathrm{~Hz}$ anti-aliasing filters. Post-processing of the signals from the THeTA was accomplished in MATLAB which included down sampling the data to $250 \mathrm{~Hz}$ to match the sampling rate of the flow measurements (discussed in \$2.4). The heat flux measurements were then divided by the timeresolved temperature difference between the sensor surface and the water to obtain the time-resolved heat transfer coefficient with an estimated uncertainty of $\pm 7 \%$ :

$$
h(t)=\frac{q^{\prime \prime}(t)}{T_{s}(t)-T_{\infty}} .
$$

\subsection{Flow measurements: time-resolved particle image velocimetry}

The present study examines the full two-dimensional velocity field in front of the flat plate using TRDPIV which delivers non-invasive flow field measurements with high spatiotemporal resolution. Details on the general method are provided by Westerweel (1997) and Raffel et al. (2007).

As shown in figure 1 the TRDPIV system used employs a New Wave Research Pegasus dual head laser. An optical train is used to focus the laser light into a thin $(\approx 1 \mathrm{~mm})$ sheet which illuminates the fluid plane containing the region of interest (ROI), located directly in front of the ten sensors. Neutrally buoyant glass microspheres with a mean diameter of $11 \mu \mathrm{m}$ were used as tracer particles in the flow. Motion of the particles was tracked from underneath with an IDT XS-5 high-speed camera using its maximum resolution of $1280 \times 1024$ pixels. With a magnification of $51 \mathrm{um} \mathrm{pixel}{ }^{-1}$, the camera interrogated an ROI $6.5 \mathrm{~cm}$ wide (along the plate) by $5.2 \mathrm{~cm}$ long (normal to the plate).

For the stagnation tests, the TRDPIV measurements were taken in a single-pulsed fashion for approximately $13 \mathrm{~s}$ at a sampling rate of $250 \mathrm{~Hz}$. For the vortex ring tests, the TRDPIV measurements were taken in a double-pulsed fashion for approximately $6.5 \mathrm{~s}$ at a sampling rate of $250 \mathrm{~Hz}$. Due to the large velocity dynamic range encountered across the different vortex ring conditions, no single pulse separation could be used across the entire set of experiments. Therefore, for each different vortex ring produced, preliminary testing was performed to determine the optimal pulse separation needed to obtain the desired particle displacements. This ranged from $2 \mathrm{~ms}$ for the slowest rings to $0.5 \mathrm{~ms}$ for the fastest. In both experiments, the images were processed using in-house-developed TRDPIV software (Eckstein, Charonko \& Vlachos 2008; Eckstein \& Vlachos 2009a,b). Images were processed using crosscorrelation with a $64 \times 64$ pixel window followed by a $32 \times 32$ window using the robust phase correlation after a discrete window offset. Using 8 by 8 pixel vector spacing, the ROI contained 19625 vectors with $416 \mu \mathrm{m}$ between vectors. The total error in TRDPIV velocity measurements using the aforementioned experimental setup and image-processing techniques was estimated to be $\pm 0.05-0.1$ pixels (Eckstein \& Vlachos 2009b).

\subsection{Vortex identification and circulation calculation}

Starting with the velocity vector fields obtained from the TRDPIV analysis, a vortex identification scheme was employed to dynamically identify the vortices present in the ROI. In this study, the swirling strength (or $\lambda_{c i}$ ) method of Zhou et al. (1999) was employed. Similar to the Delta criterion (Chong, Perry \& Cantwell 1990), the $\lambda_{c i}$ method identifies vortices as regions where the velocity gradient tensor $(\nabla u)$ has 
complex eigenvalues, indicating swirling flow. In the $\lambda_{c i}$ method, the imaginary part of the complex eigenvalue of $\nabla u$ is used to identify vortices as it is a measure of the swirling strength of the vortex. To obtain the eigenvalues, the velocity-gradient-tensor characteristic equation is solved.

The imaginary part of the complex eigenvalue then gives the swirling strength at the location in the flow for which $\nabla u$ is calculated. While in theory any non-zero value for $\lambda_{c i}$ indicates the presence of a vortex, Zhou et al. (1999) suggest setting some positive threshold at a few per cent of the maximum value. By doing so, the identification of extraordinarily weak structures is minimized. Therefore, in the present work, any location where $\lambda_{c i}$ is greater than $2 \%$ of the maximum value for the flow field is identified as a point within a vortex.

After identifying the vortical structures, the circulation of the vortex was calculated. Circulation is defined as the line integral around a closed curve of the velocity (Panton 2005):

$$
\Gamma=\oint_{C} \boldsymbol{V} \cdot \mathbf{d} \boldsymbol{l}
$$

Equation (2.2) is applied to the TRDPIV measured velocity field around the regions which are identified as vortices. The circulation is calculated through the line integral of the velocity along the closed path enclosing the identified vortical structure. The $\lambda_{c i}$ iso-contour line defining the vortex region is segmented based on the TRDPIV spatial sampling resolution and the dot product of each segment with the measured velocity is computed. The sum of all the dot products then gives the measured circulation for that particular vortex.

\subsection{Steady-state properties of the heated surface: low-turbulence stagnation baseline}

Preliminary stagnation tests were performed without the grid installed in the tunnel in order to validate the experimental approach and provide a baseline flow and heat transfer quantities for comparison with the turbulent cases. In stagnating flows, the most important parameter for both thermal and fluid analysis is the spatial acceleration parameter, $a$ where $u=a x$. This parameter was determined from the average of the measured vector fields to be $0.98 \mathrm{~s}^{-1}$ which compares well with the correlation given by White (1974) for a square body in cross-flow, which relates $a$ to the free-stream velocity $U$ and the body height $L$ :

$$
a \approx \frac{\pi}{2} \frac{U}{L}=1.03 \mathrm{~s}^{-1}
$$

The excellent agreement between the predicted and measured acceleration parameter supports its use for the calculation of other properties including the laminar boundary layer and thermal boundary layer thicknesses:

$$
\delta=2.4 \sqrt{\frac{\nu}{a}}, \quad \delta_{T}=\delta \operatorname{Pr}^{-0.4} .
$$

Using the kinematic viscosity $(v)$ and Prandtl number $(P r)$ at the film temperature gives $\delta=2.1 \mathrm{~mm}$ and $\delta_{T}=1.1 \mathrm{~mm}$. The acceleration parameter can also be used to predict the baseline heat transfer coefficient from the surface using the correlation given by Goldstein (1938):

$$
h_{0}=0.57 k \operatorname{Pr}^{0.40} \sqrt{\frac{a}{v}}
$$


where $k$ is the thermal conductivity of the fluid. Equation (2.5) predicts a baseline heat transfer coefficient of $h_{0}=740 \mathrm{~W}\left(\mathrm{~m}^{2} \mathrm{~K}\right)^{-1}$ which matches the value of $h_{0}=755 \mathrm{~W}\left(\mathrm{~m}^{2} \mathrm{~K}\right)^{-1}$ measured by the THeTA to within the experimental uncertainty. In the results shown throughout the paper, the measured convective heat transfer coefficients are expressed in dimensionless form by the Stanton number using the product of the free-stream velocity, specific heat, and density $\left(U_{\infty} C \rho\right)$ :

$$
S t=\frac{h}{\rho C_{p} U_{\infty}} .
$$

\subsection{Steady-state properties of the heated surface: natural convection}

For the vortex ring tests, the properties of the natural convection boundary layer were determined prior to the ring interaction. Both the fluid and thermal properties of the steady-state boundary layer were measured.

TRDPIV was used to measure the profile of the natural convection boundary layer. This test was performed using the TRDPIV setup described above except the laser plane was aligned vertically from below the tunnel and the camera was mounted horizontally. Also, the magnification was increased to $30 \mu \mathrm{m}$ pixel $^{-1}$ to resolve the near-wall flow field within the boundary layer. To check self-similarity, dimensionless velocity was plotted versus a dimensionless coordinate $\eta$ involving the Grashof number $G_{y}$. The dimensionless velocity is defined as:

$$
f^{\prime}(\eta)=\frac{v y}{2 v} G_{y}^{-1 / 2}
$$

and the dimensionless coordinate and Grashof number are defined as

$$
\eta=\frac{x}{y}\left(\frac{G_{y}}{4}\right)^{1 / 4}, \quad G_{y}=\frac{g \beta y^{3}\left(T_{s}-T_{\infty}\right)}{v^{2}}
$$

where $v$ and $\beta$ are the kinematic viscosity and thermal expansion coefficient of water and $g$ is the gravitational constant. The variables $y$ and $x$ are the coordinates parallel and normal to the plate respectively and $v$ is the velocity parallel to the plate. Figure 5 shows the wall jet profiles for multiple locations along the plate. A good collapse is observed and is in good agreement with the classic work by Ostrach (1953).

The thermal state of the natural convection boundary layer was also examined using the heat flux and surface temperature measurements. The heat transfer coefficient at a point on the plate a distance $y$ from the bottom can be predicted using the equation (Incropera \& DeWitt 2002)

$$
h=\frac{k}{y}{\frac{G_{y}}{4}}^{1 / 4} \frac{0.75 \operatorname{Pr}^{1 / 2}}{\left(0.609+1.221 \operatorname{Pr}^{1 / 2}+1.238 P r\right)^{1 / 4}}
$$

where $k$ and $P r$ are the thermal conductivity and Prandtl number of water evaluated at the film temperature. Plugging in the appropriate values, (2.9) predicts the heat transfer coefficient at the sensor location to be $543 \mathrm{~W}\left(\mathrm{~m}^{2} \mathrm{~K}\right)^{-1}$. The average value measured by the THeTA was $520 \mathrm{~W}\left(\mathrm{~m}^{2} \mathrm{~K}\right)^{-1}$ which is about $5 \%$ below the value predicted by (2.9), but within the experimental uncertainty.

While the thermal boundary layer thickness was not directly measured in the present study, the match between the predicted and measured properties of the natural convection momentum boundary layer allows the thermal boundary layer thickness to 


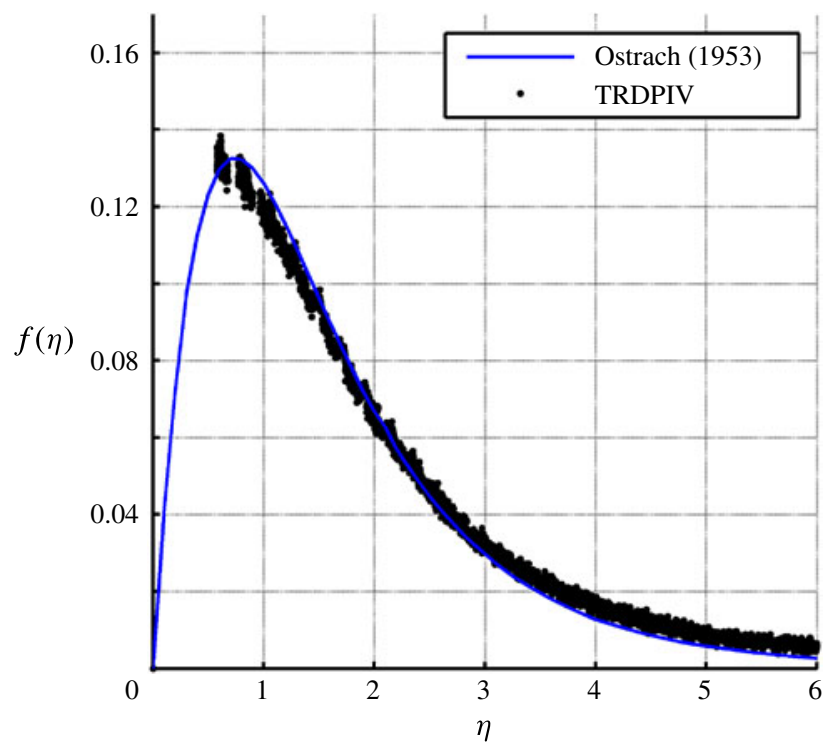

FIGURE 5. (Colour online) Non-dimensional boundary layer profile and standard scaling.

be calculated. For water, Ostrach (1953) gives the following relation:

$$
\delta_{T}=2.5 y\left(\frac{G r_{y}}{4}\right)^{-1 / 4} .
$$

This gives a value of $\delta_{T}=2.1 \mathrm{~mm}$ at the position of the heat flux sensors for the conditions of the vortex ring experiments, which matches quite well the value for the stagnation experiments. Both the natural convection and stagnation region thermal boundary layer thickness values will be used extensively throughout this paper.

\section{Experimental observations of vortex-induced heat transfer}

3.1. Vortex-wall interaction in turbulent stagnation flow and resulting heat transfer

In stagnating flows, free-stream structures are amplified into strong vortices in the nearwall region. A sequence of movies was produced for each turbulence grid showing the measured vortex interactions and resulting surface heat transfer as a function of time. A qualitative analysis of these results is most instructive to understand the basic mechanism of heat transfer augmentation.

First, figure 6 shows a representative zoomed-in view of the near-wall flow and convection at one instant of time when several vortices are in close proximity to the surface. The contour of the vector plot indicates vorticity normalized by the ratio of the turbulence-grid blockage-adjusted velocity $\left(V_{b}=V_{\infty} /(1-S)\right)$ to the bars spacing $\left(D_{b}=M-b\right)$ so that $\omega^{*}=\omega /\left(V_{b} / D_{b}\right)$. The bar plot in figure $6(b)$ represents the instantaneous convection (plotted as the Stanton number normalized by the low-turbulence baseline) at the five sensing locations shown relative to the laminar baseline. By measuring the heat transfer at the multiple sensing locations of the THeTA, the mechanism by which the vortices increase the convection is revealed. Three strong vortices with alternating rotations are observed near the surface. This arrangement of vortices produces alternating areas of downwash and upwash at the 

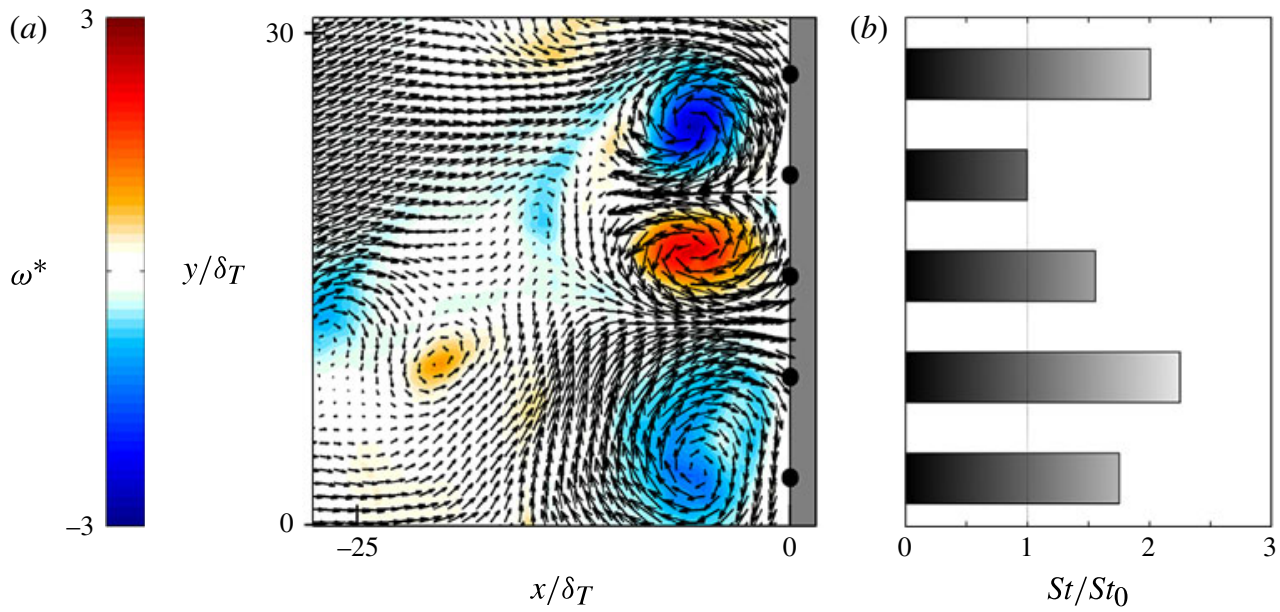

FIGURE 6. (Colour online) (a) Instantaneous snapshot of the flow field and $(b)$ normalized Stanton number. Sensor locations are indicated by the black dots in $(a)$ and convection measurements are given by the bars in $(b)$. The contour of the vector field in $(a)$ shows the normalized vorticity $\omega^{*}=\omega /\left(V_{b} / D_{b}\right)$. Convection increases are localized to areas of vortex downwash. See supplementary movie 1 available at http://dx.doi.org/10.1017/jfm.2012.589.

surface between the individual vortices. These results, in combination with the heat transfer measurements, show that the downwash regions correspond to high levels of convection since cool fluid from the free stream is being forced towards the surface in these regions. Alternatively, regions of upwash correspond to smaller increases in convection since the fluid reaching the wall has been pulled along the heated surface prior to reaching these locations. This previously warmed fluid is less effective at removing heat from the surface in these upwash regions and is similar to the observations of Sabatino \& Smith (2008) where it was observed that the highest heat transfer in turbulent spots was near the perimeter where the vortices had the nearest access to cool free-stream fluid.

A second approach to view the interaction is shown in figure 7. Here, a single vortex trajectory is shown along with the convection time histories from three nearby sensors. In figure $7(a)$, the identified vortex (with clockwise rotation) is shown over a time period of $5.3 \mathrm{~s}$. The size of the overlapping circular markers is proportional to the measured circulation of the vortex while its colour corresponds to the time at which it was identified as indicated by the colour bar in figure 7(b). The measured convection is shown in figure $7(b)$ for the three sensors whose positions are indicated by the markers in figure $7(a)$. At first, the vortex is both weak and far from the surface and has little impact on the convection over the first $2 \mathrm{~s}$. Then, the vortex moves towards the surface and its circulation is amplified. At this point, the top sensor (o) is positioned directly in the downwash region and experiences more than double the laminar baseline convection. As the vortex approaches the surface, the convection from the middle sensor (+) also increases slightly. Once the vortex is close to the surface, its mirror-image on the wall (from potential flow theory) induces a downward velocity bringing the middle sensor into the vortex downwash region. Note, however, that by the time that the middle sensor is in a true downwash region, the vortex has moved farther from the surface and weakened slightly. The middle sensor does experience an increase in convection but not to the extent of the top sensor. Finally, as the vortex 

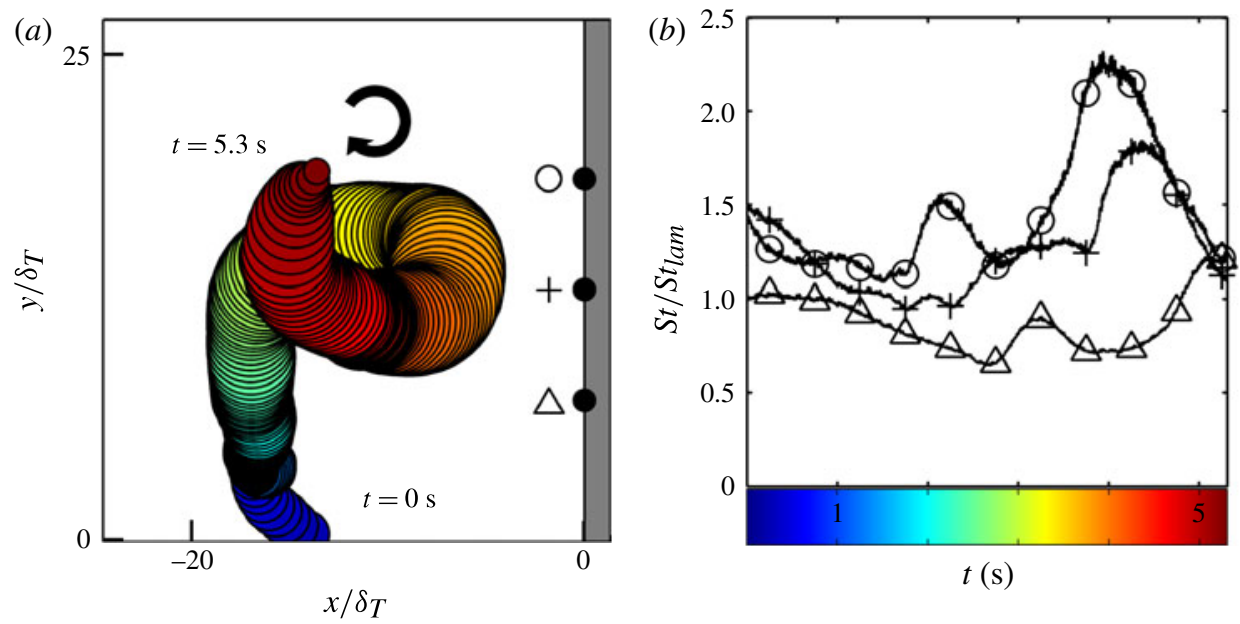

FIGURE 7. Transient interaction of a single vortex with the surface and resulting convection. Vortex location and strength are indicated by the overlapping circular markers in $(a)$ while the colour corresponds to the time in $(b)$, where the convection history for the three nearby sensor locations (marked in $a$ ) is shown.

continues to move from the surface and lose strength, the convection decays to the baseline level. Since the bottom sensor $(\triangle)$ remained in the upwash region of the vortex, its convection remained below the baseline level for nearly the entire time of the interaction.

Over 200 heat transfer events similar to the one shown in figure 7 (events in which the heat transfer coefficient increased by more than $20 \%$ ) were examined from the stagnation flow data. These ranged from short duration events lasting less than a quarter of a second to individual events lasting up to five seconds. In some cases a vortex would remain nearly stationary for a long period of time while in others the vortex would traverse the entire width of the instrumented region in under a second. Even though the nature of the thermal events was very diverse, in over $70 \%$ of the cases, the sensor measuring the thermal event was located in a region identified as the downwash region of the most dominant (largest induced velocity) local vortex.

These observations provide initial qualitative insight into the complex processes controlling the vortex-induced heat transfer. However, the complexity of a fully developed turbulent flow, with its three-dimensionality and the dynamic interactions of numerous coherent structures with each other and with the wall, does not enable deducing the role of an individual vortex in a controlled and repeatable fashion. With this in mind, a second experiment was performed which examined the simpler case of a vortex-ring-wall interaction and the ensuing heat transfer. The results of this experiment are discussed next.

\subsection{Vortex-ring-wall interaction and resulting heat transfer}

Representative results from the planar TRDPIV measurements are shown along with the corresponding heat flux measurements during the vortex-ring-wall interaction in figure 8. This is used to gain a qualitative understanding of the effect of a single vortex interaction on the surface heat flux. In figure $8(a)$, velocity fields are shown at six evenly spaced time steps over half a second with the contour representing the vorticity normalized by the ratio of the maximum piston velocity to orifice 

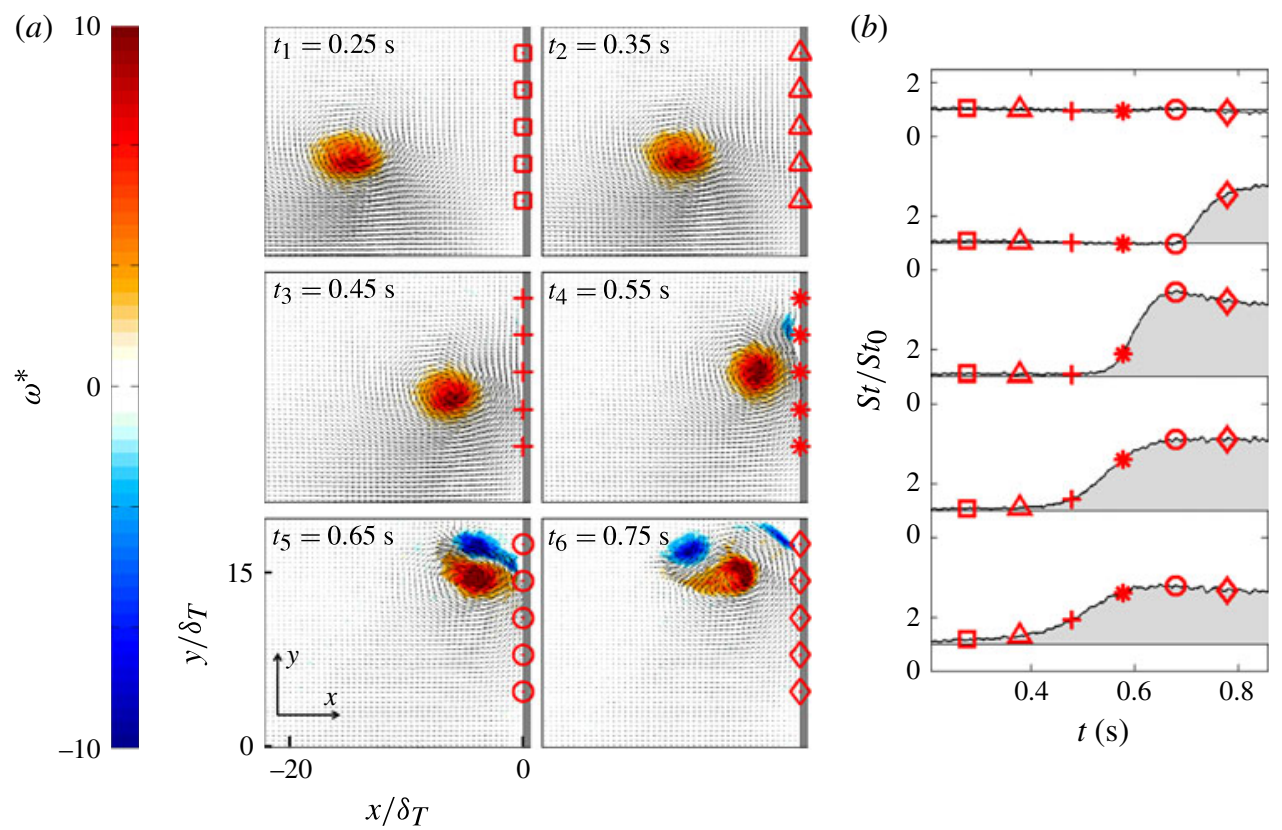

FIGURE 8. (Colour online) Illustration of ring impingement and heat transfer. (a) Velocity field sequence represent one half of the vortex ring over a half-second period. As the ring approaches the heated plate the measured convection increases as illustrated in $(b)$. Markers on the right of the velocity fields correspond to five of the individual heat flux sensor locations, and the time sequence for each of these five sensors is shown in $(b)$.

diameter, $\omega^{*}=\omega /\left(U p_{\max } / D\right)$. The velocity fields show one half of the vortex ring (the bottom axis coincides with the ring's axis of symmetry). The five markers on the right-hand side of the vector fields indicate the location of five heat flux sensors. In figure $8(b)$ measured transient Stanton numbers at these five sensor locations are shown normalized by the free convection value, $S t_{0}$. For all results from the vortex ring experiments, the measured initial propagation velocity of the vortex ring $(U)$ is used in place of $U_{\infty}$ to non-dimensionalize the heat transfer coefficient (equation (2.6)). Note that only the last velocity plot is after the vortex has rebounded from the surface. This present work is primarily focused on the time preceding the vortex rebound from the surface.

As the vortex ring approaches the heated plate its propagation velocity decreases and its radius increases as it stretches (Walker et al. 1987). In figure 8, this is observed as the vortex moving in the positive $y$-direction in the ROI, away from its axis of symmetry (the other half of the ring travels in the negative $y$-direction, not shown). As the vortex reaches the plate, vorticity of opposite sign is generated at the surface. At approximately $t=0.55 \mathrm{~s}$, an eruption (Doligalski et al. 1994) occurs and a secondary vortex forms outboard of the primary. The formation of the secondary vortex causes a rapid decrease in the radial growth of the primary ring. Consequently, the primary vortex never moves further up the plate and never passes the top sensor. The observed behaviour of the vortex ring is in qualitative agreement with the work of Walker et al. (1987).

As the vortex ring interacts with the heated plate, the convective heat transfer increases dramatically compared to the unperturbed natural convection level. 


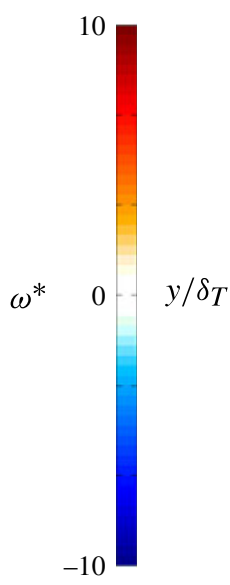

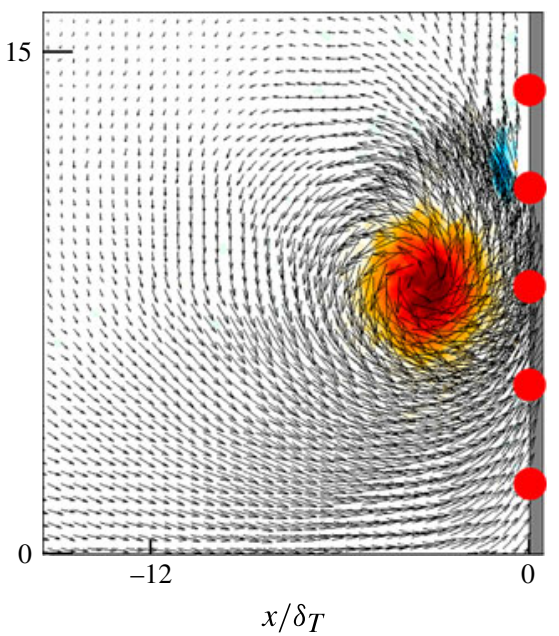

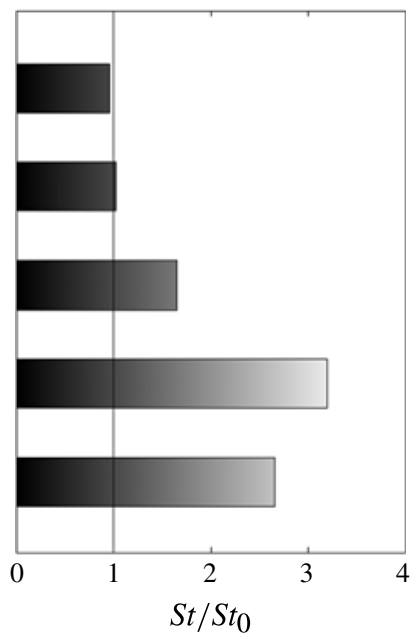

FIgURE 9. (Colour online) Mechanism of heat removal. Close-up of interaction process and measured rate of convection at one time instant. The contour of the vector field shows the normalized vorticity $\omega^{*}=\omega /\left(U p_{\max } / D\right)$. The sensors in the downwash region of the vortex experience more convection than the sensor directly beneath the vortex and much more convection compared to sensors in the upwash region.

To highlight the heat-transfer augmentation caused by the vortex interaction, the grey-shaded area in figure $8(b)$ represents the increase between the measured Stanton number and the levels corresponding to natural convection. Note that when the vortex is in close proximity to the sensor, in some instances the convective transport increases by more than a factor of four over the free convection levels. Many tests were performed where only thermal data were recorded and it was found that these high levels of localized heat transfer were consistently present and always at least double the free convection level. This is in stark contrast to the value of $15 \%$ reported from spatially averaged measurements (Arévalo et al. 2007) and illustrates that the vortex has a very localized but dominant impact on the convection.

To further demonstrate the impact that a near-surface vortex has on the local heat transfer, figure 9 illustrates the spatial convection distribution caused by a single vortex. Here, the flow field in the vicinity of the single vortex is shown with the instantaneous convection from five nearby sensors. Since the vortex is rotating counterclockwise, the two sensors above the vortex are in the upwash region where the vortex is pulling fluid away from the surface. These two sensors indicate that the convection at this point in time is essentially the natural convection level. The two sensors below the vortex are in a strong downwash region and experience a significant increase in convection. Notably, these sensors in the downwash region are experiencing significantly more convection compared to the sensor nearest to the vortex even though it is in a region of maximum shear stress (Arévalo et al. 2010). These results indicate that the outer fluid which is entrained towards the sensor has a much larger effect compared to fluid sweeping along the sensor parallel to the surface. Again, since heat has been transferred to this fluid while traversing the surface, it is ineffective at removing heat from the surface in the upwash region. 

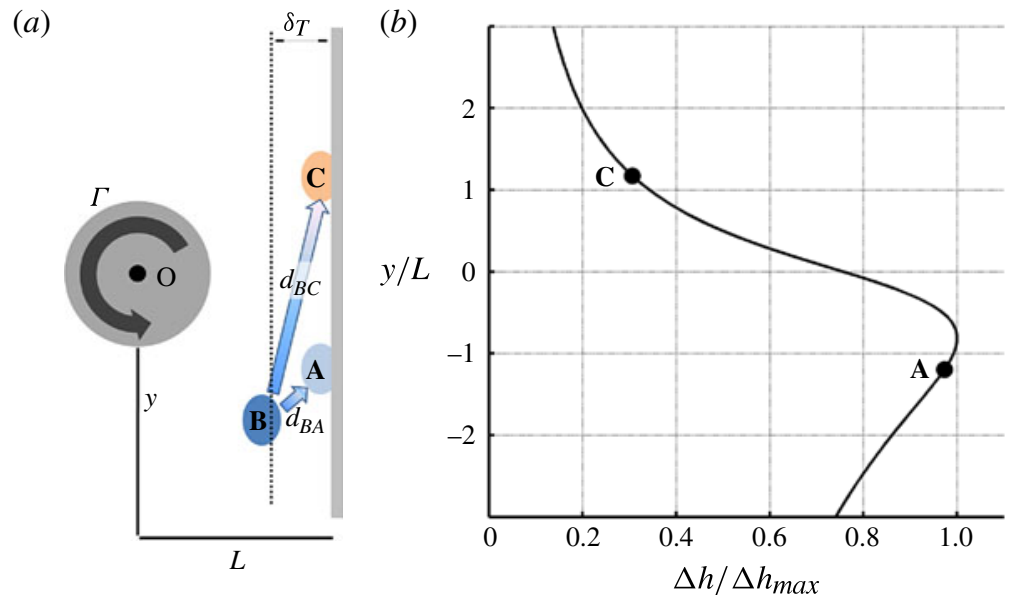

FIgURE 10. (Colour online) (a) Schematic used in model development, along with $(b)$ the augmentation prediction (equation (4.5)) as a function of non-dimensional vortex location $y / L$. Model predicts much larger convection in the downwash region compared to the upwash region due to the smaller value of $d_{B A}$ compared to $d_{B C}$.

\section{Physical model for vortex-induced transient heat transfer augmentation}

The current effort to develop a physically based model to predict the convective transport due to a vortex is based on the surface renewal model of Nix, Diller \& Ng (2007). In their work, it was hypothesized that when a flow structure penetrates through the boundary layer, interaction with the surface causes an increase (augmentation) in the rate of heat transfer over that due to transport through the laminar boundary layer. This process was modelled as a purely conductive event treating the flow structure as a semi-infinite medium. Heat is conducted to the structure for a characteristic time $\tau$ :

$$
\Delta h=\frac{\Delta q}{T_{\infty}-T_{S}}=\frac{k}{\sqrt{\pi \alpha \tau}}
$$

where $\alpha$ is the thermal diffusivity of the fluid. By defining the time scale as the ratio of the mean streamwise integral length scale to streamwise r.m.s. fluctuating velocity, this model successfully predicted experimentally measured values of increases in the time-averaged heat transfer coefficient (Nix et al. 2007).

Using the surface renewal model based on the semi-infinite conduction assumption as described above, an extension is developed which captures the time-dependent physics of the vortex-surface interaction. Consider the case of a single vortex located at distance $L$ from a heated surface with a thermal boundary layer of thickness $\delta_{T}$ as illustrated in figure 10. The vortex induces a velocity field according to the Biot-Savart law (Panton 2005) which forces outer fluid through the boundary layer. This process can be thought of as individual fluid elements which start at the outer fluid temperature $\left(T_{\infty}\right)$, move through the thermal boundary layer, and interact with the surface. As soon as the fluid element reaches the thermal boundary layer, it begins exchanging thermal energy with the warmer fluid within the boundary layer which causes the temperature of the fluid element to increase. The longer the fluid element remains within the thermal boundary layer, the more heat is transferred to it and the more its temperature rises which decreases its capacity for exchanging thermal energy 
with the surface. Consequently, the time for the fluid to travel through the thermal boundary layer is the proper characteristic time scale for the heat transfer process.

The transit time through the thermal boundary layer is dependent on two factors: the distance that the fluid element travels within the boundary layer to reach the surface $\left(d_{B A}\right)$ and the average induced velocity along this distance. Both of these values are estimated from the velocity field produced by a free vortex of specified strength at the identified location and both vary at each time step depending on the strength and location of the vortex. The average induced velocity is approximated assuming a linear drop to zero at the surface and is therefore one half the induced velocity at the edge of the thermal boundary layer (point B) calculated from the Biot-Savart law. For point A, this is:

$$
\bar{V}(t)=\frac{1}{2} V_{I N D}(t)=\frac{1}{2} \frac{\Gamma(t)}{2 \pi d_{O B}(t)} .
$$

The distance that the fluid travels within the boundary layer is the distance from the point of interest on the surface to the point where a circle, concentric with the centre of the vortex, crosses the edge of thermal boundary layer (point B), as illustrated in figure 10, where $d_{O B}$ is the distance from the vortex center to the point $B$. The characteristic time for point $\mathrm{A}$ is then:

$$
\tau(t)=\frac{d_{B A}(t)}{\bar{V}(t)} .
$$

It is expected that a vortex will not have an immediate effect on the surface convection because of the time delay to reach the surface. Therefore, it is necessary to account for the time required for the induced outer fluid to reach the surface. The appropriate time scale is the ratio of the thermal boundary layer thickness $\left(\delta_{T}\right)$ to the average induced velocity (equation (4.2)). Physically, this represents the shortest time that it would take the outer fluid to cross the thermal boundary layer:

$$
\tau_{\text {Delay }}(t)=\frac{\delta_{T}}{\bar{V}(t)} .
$$

Due to the square root in the denominator of the time-averaged augmentation model (equation (4.1)), the instantaneous model has a factor of two in the denominator relative to the time-average result in (4.1) from the integration of the square-root function. Incorporating the time delay and the factor of two, the heat transfer coefficient augmentation prediction at point A becomes:

$$
\Delta h\left(t+\tau_{\text {Delay }}(t)\right)=\frac{k}{2 \sqrt{\pi \alpha \tau(t)}} .
$$

Adding this augmentation to the heat transfer coefficient of the undisturbed boundary layer as the square root of the sum of the squares gives:

$$
h\left(t+\tau_{\text {Delay }}\right)=\sqrt{h_{0}^{2}+\Delta h\left(t+\tau_{\text {Delay }}\right)^{2}} .
$$

While the model described above is very simple, incorporating $d_{B A}$ enables it to capture the dynamics between the upwash versus the downwash effect that was observed experimentally in figures 6 and 9. This is illustrated in figure $10(b)$ by plotting the augmentation prediction (equation (4.5)) as a function of non-dimensional vortex location $y / L$ where $y$ is the distance from the surface location to the point on the surface closest to the vortex. Note that while points $\mathrm{A}$ and $\mathrm{C}$ are equidistant 

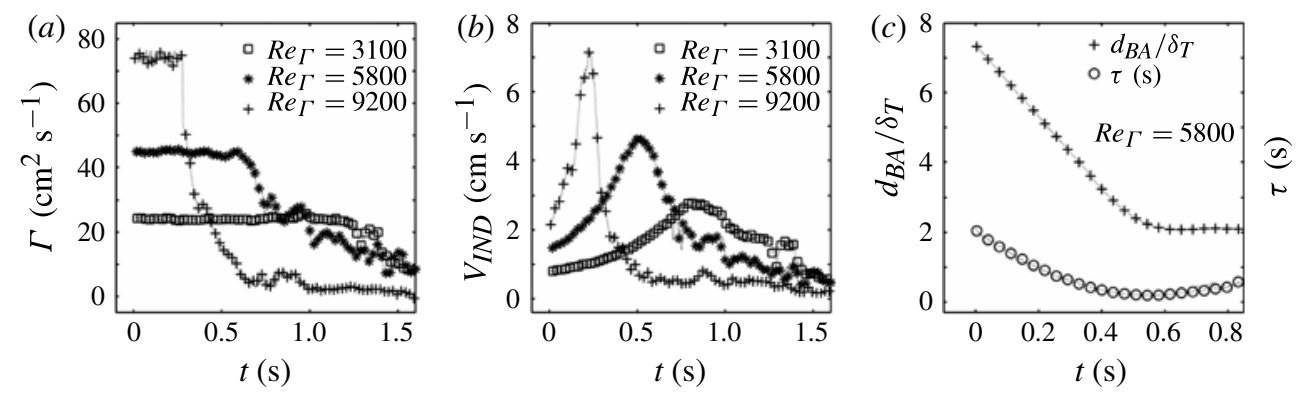

FIgURE 11. (a) The measured circulation of the vortex as it approaches the plate for three tests. (b) The circulation is used to calculate the induced velocity at the sensor location on the plate using the Biot-Savart law. (c) The distance that fluid must travel within the thermal boundary layer $\left(d_{B A},+\right)$ is calculated from the measured vortex location. Combining this value with the induced velocity from above yields the characteristic time $(\tau, \circ)$ used in the model. $R e_{\Gamma}=5800$.

from the vortex centre, point A experiences much more convection because $d_{B A}<d_{B C}$. This curve is similar in shape to the experimentally obtained values shown in figure 9 . The basic premise of the present model is the same as the one used by Nix et al. (2007) that as cold fluid is brought to the surface by a fluid disturbance it exchanges additional thermal energy, augmenting the heat transfer. Nix et al.however, used an average characteristic time based on the integral length scale of the turbulence for their surface renewal model to give the time-average added heat transfer. Our model analyses each individual event as a function of time, calculating the transit time through the boundary layer for each packet of fluid. The shorter the time, the higher the resulting heat transfer and the shorter the delay before the effect occurs at the surface. This gives the time-resolved heat flux at each location on the surface. In both models the increase is combined with the laminar or undisturbed heat transfer value.

\section{Model validation}

\subsection{Model comparison to the vortex-ring-induced heat transfer measurements}

In each vortex ring experiment, the vortex ring interacted with a number of sensors, which can all be used for comparison with the model. In all, comparisons were performed for 33 time records of velocity and heat flux spanning five different values of $R e_{\delta}$ at five different heat flux sensor positions. This section along with figures 11-13 give a detailed description of the interaction process for one representative sensor for tests corresponding to vortex ring Reynolds numbers of $R e_{\Gamma}=3100,5800$, and 9200 . The $R e_{\Gamma}=5800$ case is the same as shown in figure 8 for the fourth heat flux sensor from the top. Figure 13 then summarizes the results for all the tests.

The measured transient circulation and the induced velocity at a single sensor location are shown in figures $11(a)$ and $11(b)$ respectively for three vortex strengths spanning the range of test conditions. As the vortex ring propagates towards the plate, the circulation remains nearly constant. Then, upon reaching the plate, vorticity of opposite sign is generated at the wall and the vortex strength quickly diminishes. This is seen as the abrupt drop in circulation at $t=0.275 \mathrm{~s}, t=0.655 \mathrm{~s}$, and $t=1.39 \mathrm{~s}$ for the three tests shown. In all cases, $t=0$ corresponds to the time when the vortex ring first enters the ROI. The different times are due to the smaller propagation 


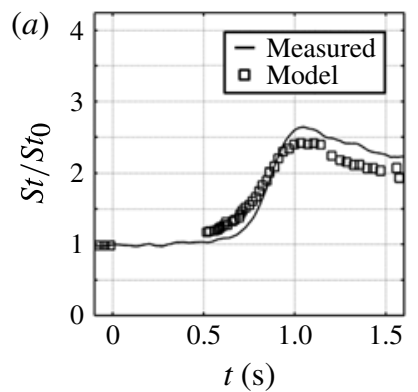

(b)

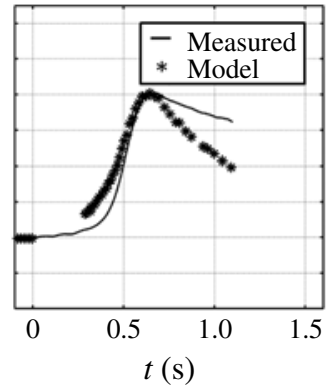

(c)

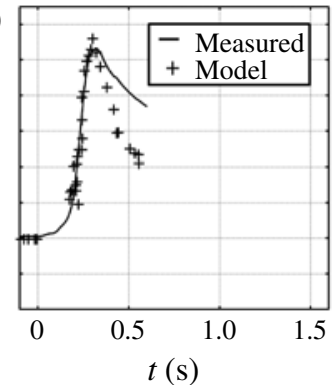

FIGURE 12. Comparison of model prediction to measured convection during vortex interaction. Results shown from three representative tests spanning the range of vortex strengths: (a) $R e_{\Gamma}=3100,(b) R e_{\Gamma}=5800,(c) R e_{\Gamma}=9200$.

velocity for the weaker vortex rings. Incorporating these measured vortex circulations with the measured vortex position, the transient induced velocity at a sensor on the plate surface is calculated for each test using the Biot-Savart law as shown in figure 11(b). Since in each test the vortex follows approximately the same trajectory, the two main differences are the speed at which the vortex passes the sensor and the induced velocity at the sensor, both of which are affected by the circulation value. Consequently, the strongest vortex with the largest $\operatorname{Re}_{\Gamma}$ causes the largest induced velocity for a short period of time while the weakest vortex causes a smaller induced velocity for a longer period of time.

The characteristic time used in the model is a function of the induced velocity and the distance that the fluid travels within the thermal boundary layer $\left(d_{B A}\right)$. Figure 11 $(c)$ shows $d_{B A}$ as a function of time for the $R e_{\Gamma}=5800$ test. Curves for the other tests are not shown due to their similarity to those shown in figure 11(c). Specifically, the curves for $d_{B A}$ are essentially identical in magnitude and are only contracted or expanded in time. The characteristic time curve is smaller in magnitude for the stronger vortices and larger for the weaker vortices. It is helpful to again examine figure 8 as $d_{B A}$ is calculated for the fourth sensor from the top for this test. At the beginning of the test, the vortex is slightly above the sensor. Therefore, the Biot-Savart law predicts that fluid interacting with the sensor travels through the thermal boundary layer at a shallow angle from beneath. Therefore, the predicted distance is more than three times the thermal boundary layer thickness. As the vortex moves closer and above, relative to the sensor, the sensor transitions to being in a downwash region and the distance approaches the minimum possible value of $\delta_{T}$ which implies that the fluid is essentially passing straight through the boundary layer, normal to the surface.

The characteristic time $(\tau)$ is then calculated by combining the curve for $d_{B A}$ with the induced velocity to produce the curve shown in figure 11(c). The time decreases as $d_{B A}$ decreases but then begins to increase at the end as the induced velocity (and circulation) decrease. The characteristic time $(\tau)$ and the time delay $\left(\tau_{\text {Delay }}\right)$ were calculated for each sensor location for each test. Plugging these values into (4.5) and (4.6) yields a prediction of the convection as a function of time at that point. Figure 12 shows a comparison of the measured and predicted convection at a single sensor location for the same data as presented in figure 11. For $t<0$, the vortex has not yet entered the TRDPIV region of interest and the prediction is therefore the natural convection (undisturbed) Stanton number, $S t_{0}$. The gap in the prediction 

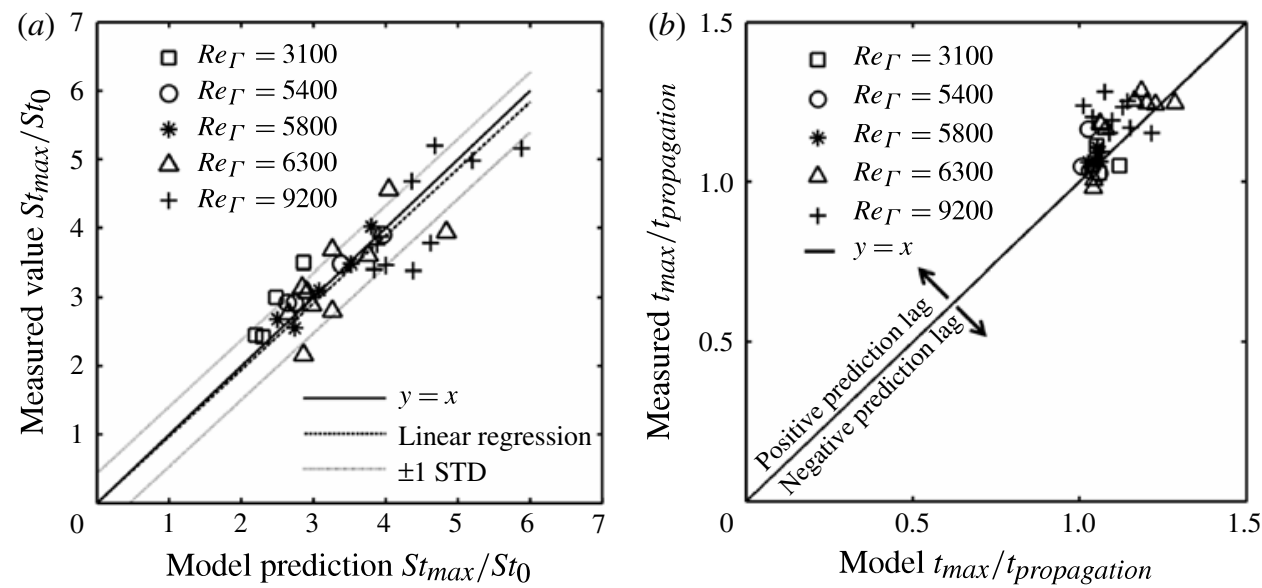

FIgURE 13. (a) Comparison of the maximum measured convection with maximum model prediction for the range of vortex ring test conditions. The bounds represent \pm 1 standard deviations from the linear regression. $(b)$ Comparison of the measured versus predicted time of maximum augmentation.

values just after $t=0$ represents the initial calculated time delay, $\tau_{\text {Delay }}$. At $t=0$, the vortex first enters the region of interest, the model is applied and combined with the natural convection Stanton number and delayed as specified. Then, as the vortex approaches the plate, the induced velocity increases and $d_{B A}$ decreases causing a shorter and shorter characteristic time which in turn causes the predicted convection to increase. Physically, the entrained fluid resides within the thermal boundary layer for increasingly shorter time.

As shown in figure 12, the transient model prediction and the measured convection curves match well in both shape and magnitude. Each different combination of $R_{\delta}$ and location produced different magnitudes of augmentation and time duration. As the vortex strength increases, the induced velocity increases causing a smaller characteristic time and smaller time delay in the model. This directly increases the augmented heat transfer coefficient predicted by (4.5).

Similar results to figure 12 were observed for all of the 33 vortex ring measured and predicted curves spanning five different test conditions and five sensor positions. To quantitatively characterize the results, the maximum values of the measured and modelled Stanton numbers are shown in figure 13(a) with the linear regression and \pm 1 standard deviation bands. In most of the cases the model predicts the measured convection within the experimental uncertainty. Using a sequential perturbation technique (Figliola \& Beasley 2000), the total estimated uncertainty $(95 \%)$ for the model prediction of the highest augmentation was calculated to be $\pm 8 \%$ for the 33 measured values. This is close to the estimated $\pm 7 \%$ uncertainty of the heat flux measurements.

It is also important that a time-resolved model predicts the correct time that the maximum augmentation occurs. This is represented in figure $13(b)$ where the time of maximum prediction is compared to the time at which the maximum augmentation was observed to occur. Since $t=0$ when the vortex first enters the TRDPIV ROI, the time is normalized by the time required for the vortex ring to propagate to the surface. As it can be seen in the figure, for the majority of cases the model is quite 

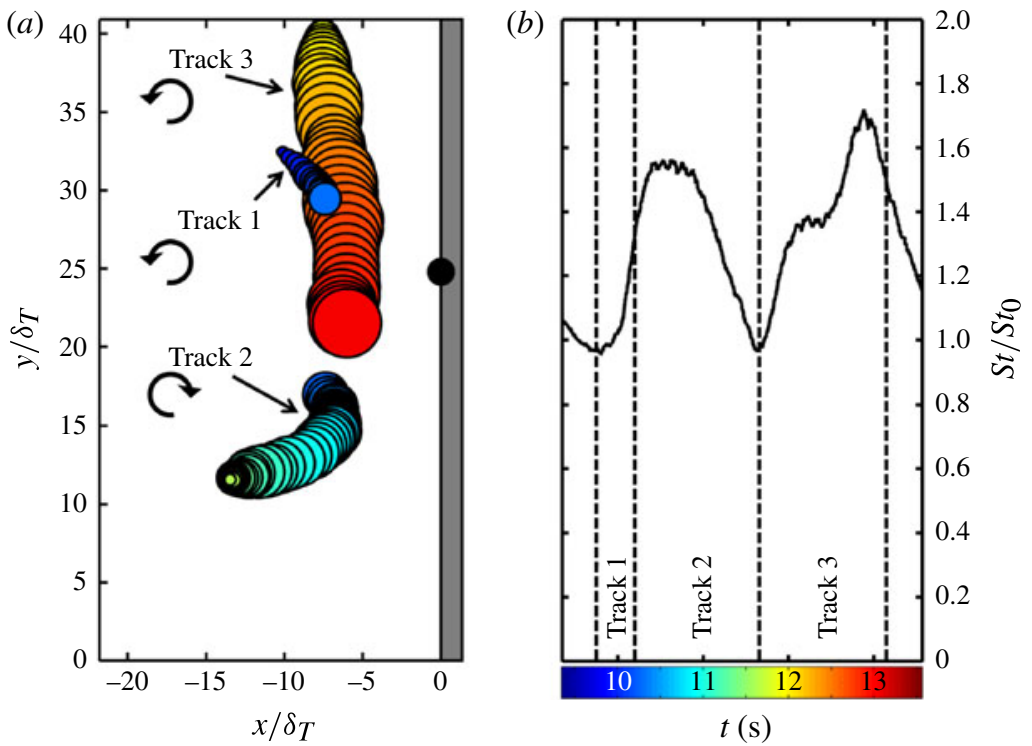

FIgURE 14. (a) Three vortex trajectories and $(b)$ resulting measured convection. Marker size in $(a)$ indicates relative strength (circulation) of vortex while the fill colour corresponds to the time as indicated in $(b)$ where the measured convection from the sensor (black dot) is shown. (b) Comparison of the measured versus predicted time of maximum augmentation.

accurate with only a small bias towards a positive time lag, namely predicting the time of maximum augmentation early. This implies that the choice for the model time delay (equation (4.4)) is appropriate.

\subsection{Model comparison to turbulent stagnation flow heat transfer measurements}

As in the previous section, the analytical model was tested by using it to predict the transient convective heat transfer coefficient at the heat flux sensor locations using the properties of the vortices identified in the measured velocity fields. For each of the turbulent cases, comparisons were made for the centre six sensors (3-8) to prevent unidentified vortices from outside the TRDPIV ROI from influencing the results. In all, 36 complete tests were used ( 3 grids $\times 2$ trials $\times 6$ sensors $)$ for the entire $13 \mathrm{~s}$ duration of the TRDPIV measurements (for a total of 117000 individual comparisons). This section along with figures 14-16 give a detailed description of the measured convection and the fluid structure during a particularly interesting set of vortex interactions from one of the tests. Table 3 then summarizes the findings from the remaining tests.

Figure 14 shows the three vortices that influence one particular sensor over the final $3.5 \mathrm{~s}$ of test number 1 of grid 2 . As in figure 7 , the marker size indicates the circulation of the vortex while its colour corresponds to the time. Figure 14(b) shows the normalized Stanton number measured by the sensor and the vertical dotted lines delineate the three tracks. The vortices shown are the ones that within the duration of the interaction dominated the near-wall region, causing the smallest calculated characteristic time, thus the largest estimated augmentation, at the sensor location at that point in time.

Examining figure 14 along with figure 15(a) gives a clear picture of the interaction process. Figure $15(a)$ shows the measured vortex properties over the same $3.5 \mathrm{~s}$ 

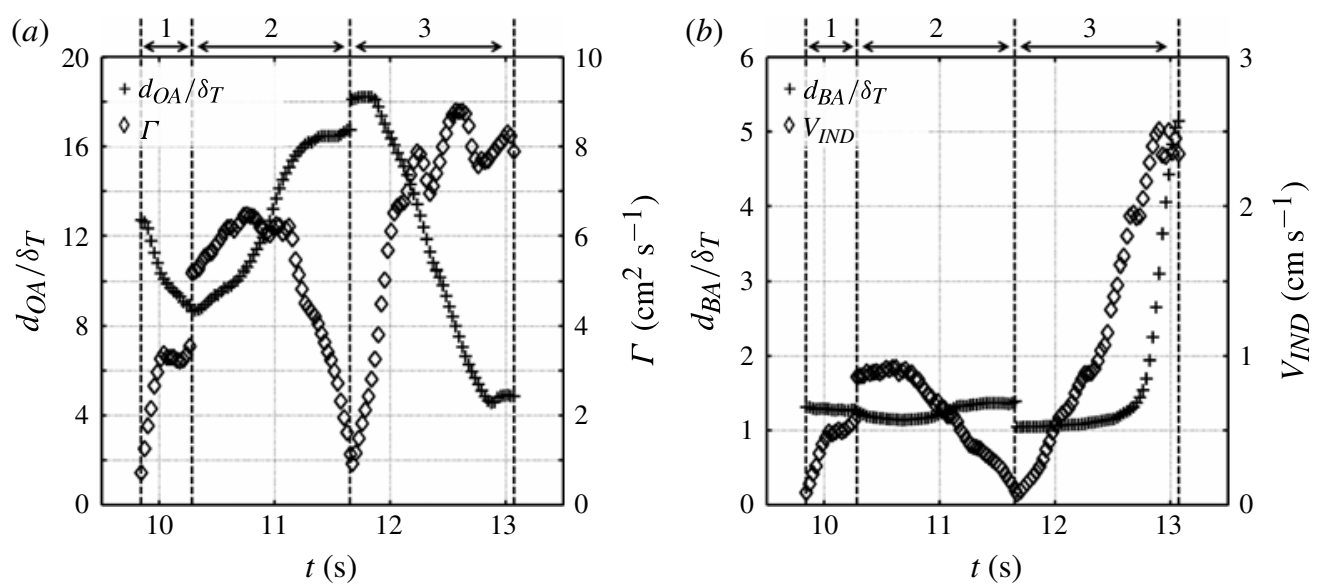

FIgURE 15. (a) Transient measured properties of the three vortices shown in figure 14 during the interaction. The measured properties are used to determine the transient model inputs as shown in $(b)$.

\begin{tabular}{|c|c|c|c|c|c|c|c|c|}
\hline \multicolumn{2}{|c|}{$\begin{array}{l}\text { Free-stream } \\
\text { turbulence }\end{array}$} & \multicolumn{2}{|c|}{$\begin{array}{l}\text { Identified } \\
\text { structures }\end{array}$} & \multicolumn{2}{|c|}{$\begin{array}{l}\text { Measured } \\
\text { convection }\end{array}$} & \multicolumn{2}{|c|}{$\begin{array}{l}\text { Modelled } \\
\text { convection }\end{array}$} & \multirow{2}{*}{$\frac{\begin{array}{c}\text { Std deviation } \\
\text { difference of } S t\end{array}}{S t(\times 1000)}$} \\
\hline Grid & $\Lambda(\mathrm{cm})$ & $\overline{\#}$ & $\overline{\left|\operatorname{Re}_{\Gamma}\right|}$ & $S t(\times 1000)$ & Aug (\%) & $S t(\times 1000)$ & Aug (\%) & \\
\hline 1 & 0.81 & 15.8 & 585 & 2.51 & 38 & 2.50 & 37 & 0.60 \\
\hline 2 & 1.93 & 8.1 & 463 & 2.35 & 29 & 2.37 & 30 & 0.54 \\
\hline 3 & 3.24 & 4.4 & 218 & 2.34 & 29 & 2.08 & 14 & 0.43 \\
\hline
\end{tabular}

TABLE 3. Summary of time-averaged results for the model applied to the stagnation data. $\overline{\#}$ denotes average number of structures and $A u g$ heat transfer augmentation.

as figure 14 and again the vertical lines delineate the three separate trajectories. Specifically, the non-dimensional distance from the vortex to the sensor (+) and the measured circulation $(\diamond)$ are shown. At the beginning of the time shown, there is no strong vortex in the vicinity of the sensor and the convection is at nearly the baseline level. At this time, the vortex which most strongly influences the convection at the sensor location is approximately 13 boundary layer thicknesses away and has a circulation of less than $1 \mathrm{~cm}^{2} \mathrm{~s}^{-1}$. This vortex then grows in strength as it approaches the stagnation region (and the sensor) with a corresponding increase in measured convection. Then, at $t=10.25 \mathrm{~s}$, a more dominant vortex emerges. Although vortex number 2 starts at approximately the same distance from the sensor as vortex number 1 , it is significantly stronger with a circulation of $5 \mathrm{~cm}^{2} \mathrm{~s}^{-1}$ compared to $3.5 \mathrm{~cm}^{2} \mathrm{~s}^{-1}$. This clockwise rotating vortex forces fluid straight through the thermal boundary layer towards the sensor, increasing the convection to almost $60 \%$ above baseline levels. Then, as the vortex begins to move away and weaken, the convection returns to the baseline level. At $t=11.7 \mathrm{~s}$, the third structure appears and quickly grows into a dominant vortex. The counter-clockwise vortex forms above the sensor then moves downward along the surface. As the vortex approaches and eventually passes the sensor, its circulation grows to a maximum of $9 \mathrm{~cm}^{2} \mathrm{~s}^{-1}$. This causes the convection 
to spike at a value nearly $75 \%$ higher than the baseline just as the vortex reaches the sensor. Then, as the sensor begins to enter the upwash region of the vortex, a drastic decrease in convection is observed even though the vortex is still quite close to the sensor. Fluid elements that reach the surface in upwash regions have resided for considerable time within the thermal boundary layer since they must now flow along the surface first. These fluid elements have therefore warmed significantly and are much less effective at convecting heat from the surface, as indicated by the sensor for $t>12.9 \mathrm{~s}$.

From the measured vortex properties, the transient model inputs were calculated as shown in figure $15(b)$. The calculated induced velocity is shown $(\diamond)$ along with the non-dimensional distance that the fluid must travel within the thermal boundary layer $(+)$. When the fluid travels a short distance within the thermal boundary layer (values of $d_{B A}$ near one), the sensor is in the downwash region and the fluid is travelling nearly perpendicular to the surface. Larger values indicate that the fluid is sweeping along the surface prior to reaching the sensor location. Again, it is assumed that the time that the fluid remains within the thermal boundary layer $(\tau)$ is simply the distance that fluid travels within the thermal boundary layer $\left(d_{B A}\right)$ divided by the average velocity that the fluid maintains along this distance $\left(V_{I N D} / 2\right)$ which is a measure of both the strength and proximity of the vortex (equation (4.2)). At $t=10.6 \mathrm{~s}$, the induced velocity is at a local maximum and $d_{B A}$ is at a local minimum which means that $\tau$ is a local minimum. Fluid, therefore, is quickly travelling a short distance to reach the surface and little heat is transferred to the fluid prior to reaching the surface. Note that in figure 14, $t=10.6 \mathrm{~s}$ corresponds to a local maximum in the measured convection. Then, as the vortex decays, $V_{I N D}$ falls to essentially zero prior to the arrival of the third vortex. In the third vortex trajectory, the effect of upwash versus downwash is most visible. For most of the interaction with the wall, the vortex is above the sensor and, since the vortex has counter-clockwise rotation, the sensor remains in a region of downwash. Once the vortex passes the sensor however, the sensor enters the upwash region. After this occurs, fluid enters the thermal boundary layer far below the sensor and must travel upwards along the surface prior to reaching the sensor as indicated by the large value for $d_{B A}$ (and a correspondingly large value of $\tau$ ). This fluid has ample time to warm as it slides along the surface and the heat transfer upon its arrival to the surface is reduced.

In figure 16, the final model prediction is shown compared to the measured convection. The shaded region indicates the $3.5 \mathrm{~s}$ discussed in the previous two figures and the vertical dotted lines again delineate the different trajectories. The dominant vortex at the sensor location is used at each instant of time with the corresponding time delay of the model included. As in the vortex ring tests, the transient model prediction matches the measured convection well in terms of both shape and magnitude. The two distinct spikes in the measured convection both correspond to times when fluid is quickly reaching the sensor from outside the thermal boundary layer. Times of low convection occur either when fluid is passing very slowly through the thermal boundary layer (weak and/or distant vortex) or when fluid must travel a great distance within the thermal boundary layer (upwash region of vortex).

Time-averaged results were obtained by integrating all of the model predictions (as illustrated in figure 16) over the time of each test. The results for all the tests are shown in table 3 . The value for each grid represents the average of 19000 individual points during the experimental tests. In addition, the standard deviation between the measured heat flux values and those calculated from the model is listed for all of 


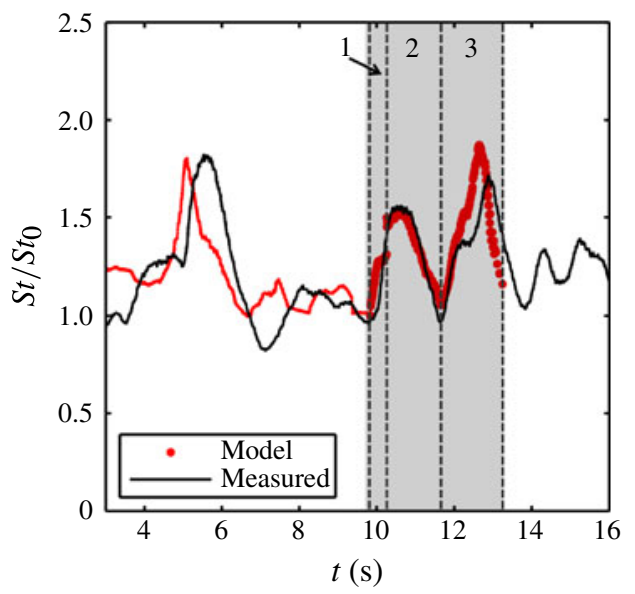

FIGURE 16. (Colour online) Model prediction compared with measured convection. The shaded region corresponds to the $3.5 \mathrm{~s}$ studied in detail in figures 14 and 15 .

the points. Because these include the variations in the magnitude of the heat flux and the delay time of the points, the values are reasonable. Clearly, averaging the values eliminates much of this variation and gives a better overall match.

As in previous studies, the measured augmentation decreases with increasing turbulence length scale. This is also the case for the model developed by Nix et al. (2007), although their overall time-averaged magnitude of augmentation was higher by a factor of two to three when applied to these data. Their model was based on experiments in air, which has very different thermal properties from the water used here. The effect of this difference could be addressed in future research. Examining the properties of the structures present gives insight into why the increase in heat transfer occurs. For each test, the average number and average circulation of the vortices identified was calculated. The average number of vortices in the TRDPIV ROI at any given time drops from nearly 16 for the shortest length scale tests to 4.4 for the longest length scale. The average magnitude of the circulation for these vortices also drops from over 4 to approximately $1.5 \mathrm{~cm}^{2} \mathrm{~s}^{-1}$. Not only are there fewer vortices available to increase the convection, but the ones that are present are much weaker and therefore have a smaller impact.

Since the model works well in predicting the convection during individual vortex interactions, it is expected to provide an accurate estimate of the time-averaged turbulent heat flux augmentation. The augmentation results shown in table 3 are calculated based on the laminar baseline value of $h_{0}=755 \mathrm{~W}\left(\mathrm{~m}^{2} \mathrm{~K}\right)^{-1}, S t_{0}=$ $1.806 \times 10^{-3}$. The model accurately predicts the measured heat transfer coefficient, with particularly good results for the smaller two length scales. Results for the tests with grid 3 did not compare as favourably and the model under-predicted the augmentation. For these tests (grid 3) both the average number (\#) and intensity of the structures identified were far lower than the grid 2 tests but the measured augmentation was similar. The grid 1 tests had many more and stronger vortices identified compared to the other tests, with a corresponding increase in both the measured and predicted augmentation. It is believed that the discrepancy between the model prediction and the measured augmentation in the grid 3 tests is due to structures which are oriented such that they are not captured by the TRDPIV measurement plane. For the grid 1 
and 2 tests, the in-plane structures are so strong that any out-of-plane motions do not significantly influence the surface convection. This is not the case for the grid 3 tests where the in-plane structures are not nearly so dominant.

\section{Conclusions}

The results presented in this work clearly demonstrate that the interaction of vortices with a surface is the primary mechanism by which heat transfer is augmented in turbulent stagnation flows. Observations of the simultaneous flow field and surface heat flux have allowed the physical mechanism of this interaction to be understood. In regions where vortices force fluid directly through the boundary layer, the convection is high because the fluid quickly reaches the surface. Conversely, regions where fluid is swept along the surface experience less convection because the fluid has already been heated within the boundary layer prior to reaching the surface. Incorporation of these observations into a first-principles-based analytical model allows accurate prediction of the time-resolved heat transfer augmentation from a single vortex as it impacts on the surface. For this simplified case, a vortex ring serves as a good model for the interaction of a single vortical structure in turbulence. For the case of free-stream turbulence in the stagnation region, the heat transfer is the combination of the heat transfer resulting from the vortex interaction and the laminar level when no free-stream turbulence is present. The results show that this method works quite well, with particularly good results obtained for the smaller length scales where the vortices are most dominant. However, more experimentation is needed to determine the true range of applicability. Specifically the experiments should be done in different fluids (most importantly air) to determine how the Prandtl number affects the results. The success of the model indicates that it is a good physical interpretation of the mechanism of heat transfer augmentation by vortices in turbulent flows. Its simplicity and the ease with which it can be implemented make the model results all the more encouraging. By looking at turbulent heat transfer as the combination of many simple vortex-wall interactions, the inherent complexity of the problem is reduced. Therefore, if the formation and motion of these large-scale structures can be accurately predicted (using LES for instance) then the resulting surface convection can be modelled without trying to solve for the fine-scale motions in the near-wall region.

\section{Acknowledgements}

The authors would like to thank Dr A. Gifford for his work developing and characterizing the turbulence grids and stagnation model. This material is based upon work supported by the National Science Foundation under grant no. CTS-0423013. Any opinions, findings, and conclusions or recommendations expressed in this material are those of the authors and do not necessarily reflect the views of the National Science Foundation.

\section{Supplementary movie}

Supplementary movie are available at http://dx.doi.org/10.1017/jfm.2012.589.

\section{REFERENCES}

Adrian, R. J., Christensen, K. T. \& LiU, Z.-C. 2000 Analysis and interpretation of instantaneous turbulent velocity fields. Exp. Fluids 29 (3), 275-290.

AmES, F. E. 1997 The influence of large-scale high-intensity turbulence on vane heat transfer. Trans. ASME: J. Turbomach. 119 (1), 23-30. 
Araya, G., Leonardi, S. \& CAstillo, L. 2008 Numerical assessment of local forcing on the heat transfer in a turbulent channel flow. Phys. Fluids 20, 085105.

Arévalo, G., Hernandez, R. H., Nicot, C. \& Plaza, F. 2007 Vortex ring head-on collision with a heated vertical plate. Phys. Fluids 19, 083603.

Arévalo, G., Hernández, R. H., Nicot, C. \& Plaza, F. 2010 Particle image velocimetry measurements of vortex rings head-on collision with a heated vertical plate. Phys. Fluids 22, 053604.

Baines, W. D. \& Peterson, E. G. 1951 An investigation of flow through screens. Trans. ASME: J. Heat Transfer 73, 467-480.

Chong, M. S., Perry, A. E. \& CAntwell, B. J. 1990 A general classification of 3-dimensional flow-fields. Phys. Fluids A 2 (5), 765-777.

Doligalski, T. L., Smith, C. R. \& Walker, J. D. A. 1994 Vortex interactions with walls. Annu. Rev. Fluid Mech. 26 (1), 573-616.

Dullenkopf, K. \& MAYle, R. E. 1995 An account of free-stream-turbulence length scale on laminar heat-transfer. Trans. ASME: J. Turbomach. 117 (3), 401-406.

Eckstein, A. C., Charonko, J. \& Vlachos, P. 2008 Phase correlation processing for DPIV measurements. Exp. Fluids 45 (3), 485-500.

Eckstein, A. \& Vlachos, P. P. $2009 a$ Assessment of advanced windowing techniques for digital particle image velocimetry (DPIV). Meas. Sci. Technol. 20 (7), 075402.

Eckstein, A. C. \& Vlachos, P. $2009 b$ Digital particle image velocimetry (DPIV) robust phase correlation. Meas. Sci. Technol. 20, 14.

EWING, J. A. 2006 Development of a direct-measurement thin-film heat flux array. PhD thesis, Blacksburg.

Figliola, R. S. \& BeAsley, D .E. 2000 Theory and Design for Mechanical Measurements, 3rd edn. John Wiley \& Sons.

Gifford, A. R., Diller, T. E. \& Vlachos, P. P. 2011 The physical mechanism of heat transfer augmentation in stagnating flows subject to freestream turbulence. J. Heat Transfer 133 (2), 021901.

Goldstein, S. 1938 Modern Devlopments in Fluid Dynamics, vol. 2. Dover, 632.

HolmberG, D. G. \& Diller, T. E. 2005 Simultaneous heat flux and velocity measurements in a transonic turbine cascade. Trans. ASME: J. Turbomach. 127 (3), 502.

Hubble, D. O. \& Diller, T. E. $2010 a$ A hybrid method for measuring heat flux. Trans. ASME: J. Heat Transfer 132 (3), 031602.

Hubble, D. O. \& Diller, T. E. $2010 b$ Development and evaluation of the time-resolved heat and temperature array. ASME J. Therm. Sci. Engng Appl. 2.

Hutchins, N. \& MARUSIC, I. 2007 Evidence of very long meandering features in the logarithmic region of turbulent boundary layers. J. Fluid Mech. 579, 1-28.

InCRoperA, F. \& DEWitT, D. 2002 Introduction to Heat Transfer, 5th edn John Willey \& Sons.

KATAOKA, K. 1990 Impingement heat-transfer augmentation due to large-scale eddies, In Proceedings of the 9th International Heat Transfer Conference, vol. l, KN-l5, pp. 255-273.

Kestin, J. 1966 The effect of free stream turbulence on heat transfer rates. Adv. Heat Transfer 3, $1-32$.

LOWERY, G. W. \& VACHON, R. I. 1975 Effect of turbulence on heat-transfer from heated cylinders. Intl J. Heat Mass Transfer 18 (11), 1229-1242.

MARTIN, R. \& ZENIT, R. 2008 Heat transfer resulting from the interaction of a vortex pair with a heated wall. Trans. ASME: J. Heat Transfer 130 (5), 051701.

Marusic, I., Mathis, R. \& Hutchins, N. 2010 Predictive model for wall-bounded turbulent flow. Science 329 (5988), 193-196.

Mayle, R. E., Dullenkopf, K. \& Schulz, A. 1998 The turbulence that matters. Trans. ASME: J. Turbomach. 120 (3), 402-409.

Moss, R. W. \& Oldfield, M. L. G. 1996 Effect of free stream turbulence on flat-plate heat flux signals: Spectra and eddy transport velocities. Trans. ASME: J. Turbomach. 118 (3), 461-467.

Nix, A. C., Diller, T. E. \& NG, W. F. 2007 Experimental measurements and modelling of the effects of large-scale free stream turbulence on heat transfer. J. Trans. ASME: Turbomach. 129 (3), 542-550. 
Ostrach, S. 1953 An analysis of laminar free-convection flow and heat transfer about a flat plate parallel tot he direction of the generating body force. Report 1111. National Advisory Committee for Aeronautics.

PANTON, R. 2005 Incompressible Flow, 3rd edn. Wiley.

Pavlova, A. \& Amitay, M. 2006 Electronic cooling using synthetic jet impingement. Trans ASME: J. Heat Transfer 128, 897-907.

Raffel, M., Willert, C., Wereley, S. \& Kompenhans, J. 2007 Particle Image Velocimetry. Springer.

Romero-Mendez, R., Sen, M., Yang, K. T. \& McClain, R. L. 1998 Enhancement of heat transfer in an inviscid-flow thermal boundary layer due to a Rankine vortex. Intl J. Heat Mass Transfer 41 (23), 3829-3840.

Sabatino, D. R. \& Smith, C. R. 2008 Turbulent spot flow topology and mechanisms for surface heat transfer. J. Fluid Mech. 612, 81-105.

Shariff, K. \& LeOnARd, A. 1992 Vortex rings. Annu. Rev. Fluid Mech. 24, U235-U279.

SUTERA, S. 1965 Vorticity amplification in stagnation-point flow and its effect on heat transfer. J. Fluid Mech. 21, 513-534.

Vanfossen, G. J. \& Simoneau, R. J. 1987 A study of the relationship between free-stream turbulence and stagnation region heat-transfer. Trans. ASME: J. Heat Transfer 109 (1), $10-15$.

Vanfossen, G. J., Simoneau, R. J. \& Ching, C. Y. 1995 Influence of turbulence parameters, Reynolds-number, and body shape on stagnation-region heat-transfer. Trans. ASME: J. Heat Transfer 117 (3), 597-603.

Walker, J. D. A., Smith, C. R., Cerra, A. W. \& Doligalski, T. L. 1987 The impact of a vortex ring on a wall. J. Fluid Mech. 181, 99-140.

WesterweEl, J. 1997 Fundamentals of digital particle image velocimetry. Meas. Sci. Technol. 8 (12), 1379-1392.

White, F. M. 1974 Viscous Fluid Flow, 3rd edn. pp. 598-599 McGraw-Hill Science/Engineering/Math.

Xiong, Z. M. \& LELE, S. K. 2007 Stagnation-point flow under free stream turbulence. J. Fluid Mech. 590, 1-33.

YUAN, T. D. \& LiBURDY, J. A. 1992 Application of a surface renewal model to the prediction of heat-transfer in an impinging jet. Intl J. Heat Mass Transfer 35 (8), 1905-1912.

Zhou, J., Adrian, R. J., Balachandar, S. \& Kendall, T. M. 1999 Mechanisms for generating coherent packets of hairpin vortices in channel flow. J. Fluid Mech. 387, 353-396. 